\title{
Inhibition of autophagic flux by ROS promotes apoptosis during DTT-induced ER/oxidative stress in HeLa cells
}

\author{
XI-YAN XIANG ${ }^{1}$, XIAO-CHUN YANG ${ }^{2}$, JIN SU $^{1}$, JING-SONG KANG $^{1}$, YAO WU $^{1}$, \\ YA-NAN XUE ${ }^{1}$, YU-TONG DONG ${ }^{1}$ and LIAN-KUN SUN ${ }^{1}$ \\ ${ }^{1}$ Department of Pathophysiology, Basic College of Medicine, Jilin University, Changchun, Jilin 130021; \\ ${ }^{2}$ Centre of Nephrology and Urology, Shenzhen University Health Science Centre, Shenzhen 130021, P.R. China
}

Received October 15, 2015; Accepted November 24, 2015

DOI: $10.3892 /$ or.2016.4725

\begin{abstract}
As targets for cancer therapy, endoplasmic reticulum (ER) stress and autophagy are closely linked. However, the signaling pathways responsible for induction of autophagy in response to ER stress and its cellular consequences appear to vary with cell type and stimulus. In the present study, we showed that dithiothreitol (DTT) induced ER stress in HeLa cells in a time- and dose-dependent fashion. With increased ER stress, reactive oxygen species (ROS) production increased and autophagy flux, assessed by intracellular accumulation of LC3B-II and p62, was inhibited. N-acetyl-L-cysteine (NAC), a classic antioxidant, exacerbated cell death induced by $3.2 \mathrm{mM}$ of DTT, but attenuated that induced by $6.4 \mathrm{mM}$ DTT. Low cytotoxic doses of DTT transiently activated c-JNU $\mathrm{N}$-terminal kinase (JNK) and p38, whereas high dose of DTT persistently activated JNK and p38 and simultaneously reduced extracellular signal-regulated kinase (ERK) activity. Combined treatment with DTT and U0126, an inhibitor of ERK upstream activators mitogen-activated protein kinase (MAPK) kinase 1 and 2 (MEK1/2), blocked autophagy flux in HeLa cells. This effect was similar to that caused by a combination of DTT and chloroquine (CQ). These data suggested that insufficient autophagy was accompanied by increased ROS production during DTT-induced ER stress. ROS appeared to regulate MAPK signaling, switching from a pro-survival to a pro-apoptotic signal as ER stress increased. ERK inhibition by ROS during severe ER stress blocked autophagic flux. Impaired autophagic flux, in turn, aggravated ER stress, ultimately leading to cell death. Taken together, our data provide the first reported evidence that ROS may control cell fate through regulating the MAPK pathways and autophagic flux during DTT-induced ER/oxidative stress.
\end{abstract}

Correspondence to: Dr Lian-Kun Sun, Department of Pathophysiology, Basic College of Medicine, Jilin University, 126 Xinmin Street, Changchun, Jilin 130021, P.R. China

E-mail: sunlk@jlu.edu.cn

Key words: endoplasmic reticulum stress, autophagy, apoptosis, reactive oxygen species, mitogen-activated protein kinase

\section{Introduction}

The endoplasmic reticulum (ER) is an essential organelle responsible for protein maturation and quality control and is the primary site of oxidative protein folding. During dysfunctional oxidative protein folding in the ER, misfolded proteins accumulate in the lumen accompanied by increased reactive oxygen species (ROS) production, a condition known as 'ER/oxidative stress' (1). Several studies have indicated that induction of ER stress is closely associated with oxidative stress during the pathogenesis of multiple human diseases such as diabetes, neurodegenerative diseases and cancer (2-5). However, the role of ROS and related signals during ER stress is not well understood.

Though the mammalian response to stress is complex, often involving multiple signaling pathways acting in concert to influence cell fate, mitogen activated protein kinase (MAPK) family members are crucial for cell maintenance. There are three types of MAP kinases, the extracellular signal-regulated kinases (ERKs), c-Jun N-terminal kinases (JNKs) and p38. Activating different combinations of MAPKs can produce distinct biological responses. JNK and p38 MAPK are activated by a diverse array of ER stress-inducing agents such as thapsigargin, tunicamycin or dithiothreitol (DTT), which may cause depletion of $\mathrm{ER} \mathrm{Ca}^{2+}$, inhibition of $\mathrm{N}$-linked protein glycosylation or impairment of disulfide bond formation (6). Sustained activation of JNK and p38 is dependent on apoptosis signaling regulating kinase 1 (ASK1), a molecule implicated in the apoptosis mediated by ER stress (7). Inhibition of MEK blocks glucose-regulated protein-78 (GRP78) upregulation and enhances apoptosis induced by ER stress in gastric cancer cells (8). In another study, the ER stress response modulated the balance between ERK and JNK signaling pathways to prevent cell death after oxidative injury (9). In addition, ROS may influence cell fate by regulating MAPK signaling pathways (10). These findings imply that ROS produced during ER stress may play a vital role, via regulation of MAPKs, in determining whether cells survive.

Autophagy is a conserved cellular protein degradation process (11) and it has been reported that ER stress is one of its major inducers (12). Most evidence indicates that autophagy activation during ER stress primarily promotes survival, clearing unfolded proteins that have accumulated in 
the ER lumen (13). However, various studies have suggested that autophagy switches from an anti-apoptotic to a proapoptotic process with increased ER stress (14). The molecular mechanisms involved in autophagy induction by ER stress are diverse, including the inositol requiring enzyme 1- $\alpha$ (IRE1$\alpha$ )-JNK and the [RNA-dependent protein kinase (PKR)-like ER kinase (PERK)]-activating transcription factor 4 (ATF4) pathways (15). In other studies, ROS regulated autophagy through MAPK signaling pathways (16). JNK activation regulated expression of autophagy-related genes $(17,18)$ and ERK activation influenced the fusion of autophagosomes and lysosomes (19). The protein p38 itself was implicated as a substrate of the autophagy pathway (6). Overall, these findings suggest that MAPK signaling pathways are involved in the process of autophagy occurring during ER stress.

The aim of the present study was to explore effects of ROS and MAPK signaling pathways on autophagy and apoptosis during DTT-induced ER/oxidative stress. Our findings suggested that, in HeLa cells, ROS was switched from a prosurvival to a pro-apoptotic signal as ER stress increased. The activation profile of MAPKs signaling pathways changed as ROS production increased, resulting in inhibition of autophagy flux. Impaired autophagic flux, in turn, aggravated ER stress, ultimately leading to cell death. Regulation of autophagy through ROS and MAPKs during ER/oxidative stress suggests novel targets for cancer therapy.

\section{Materials and methods}

Reagents and antibodies. 1,4-DTT, N-acetyl-L-cysteine (NAC), Hoechst 33258, acridine orange (AO) and 3-(4,5-dimethylthiazol-2-yl)-2,5-diphenyltetrazolium bromide (MTT) were purchased from Sigma (St. Louis, MO, USA). LysoTracker Red DND-99, fetal bovine serum (FBS), Roswell Park Memorial Institute (RPMI)-1640 culture medium, penicillin, streptomycin, propidium iodide (PI) and Annexin V-FITC were purchased from Invitrogen (Carlsbad, CA, USA). 5-(6)-carboxy-2',7'-dichlorofluorescein diacetate (DCFH-DA) was purchased from Sigma Chemical Co. (St. Louis, MO, USA). Antibodies against $\beta$-actin, Grp78, Chop, caspase-3, cleaved-caspase-3, p62, LC3-II, JNK, phospho-JNK, p38, phospho-p38, ERK and phosphor-ERK were purchased from Santa Cruz Biotechnology (Santa Cruz, CA, USA). Enhanced chemiluminescence (ECL) reagents were from Thermo Scientific (Rockford, IL, USA).

Cell culture. Human cervical carcinoma cells (HeLa cells) were purchased from the American Type Culture Collection (ATCC; Rockville, MD, USA) and cultured in RPMI-1640 medium supplemented with $10 \% \mathrm{FBS}, 100 \mathrm{U} / \mathrm{ml}$ penicillin and $100 \mathrm{mg} / \mathrm{ml}$ streptomycin (complete medium) and were grown in a humidified cell culture incubator equilibrated with $95 \%$ air and $5 \% \mathrm{CO}_{2}$ at $37^{\circ} \mathrm{C}$.

Cell viability assays. Cells were plated into 96-well plates at a density of $1 \times 10^{4}$ cells/well in $200 \mu \mathrm{l}$ complete medium. Each treatment was repeated in six separate wells. MTT reagent [ $10 \mu \mathrm{l}$ of $10 \mathrm{mg} / \mathrm{ml}$ MTT in phosphate-buffered saline (PBS)] was added to each well and cells were returned to the cell culture incubator for $4 \mathrm{~h}$. After incubation, the formazan crystals were dissolved in $150 \mu 1$ dimethylsulfoxide (DMSO). Absorbance was recorded at a wavelength of $490 \mathrm{~nm}$.

Apoptosis analysis by Hoechst 33258 staining. Apoptotic morphological alterations in nuclear chromatin were detected by Hoechst 33258 staining. Briefly, HeLa cells were cultured in 24-well plates and treated as indicated for $24 \mathrm{~h}$. Cells were washed with ice-cold PBS and fixed with $4 \%$ (w/v) paraformaldehyde overnight. The plates were then incubated with $10 \mu \mathrm{M}$ Hoechst 33258 staining solution for $10 \mathrm{~min}$. The cells were visualized under a fluorescence microscope (IX-71; Olympus, Tokyo, Japan).

Morphological assessment. Cells were seeded at $2.0 \times 10^{5}$ cells/well in 6-well cell culture dishes and treated with experimental conditions, as indicated in the individual images, during their logarithmic growth phase. After various treatments and time points, morphological alterations were analyzed under an inverted phase contrast microscope (Olympus) at a magnification of $\mathrm{x} 20$.

Western blotting. After exposure to experimental conditions, expression of selected proteins in each sample was determined by western blotting. Briefly, after quantitating protein in each sample with the Bio-Rad protein reagent (40 $\mu \mathrm{g}$ protein/well) they were separated by SDS-PAGE and transferred onto an Immun-Blot PVDF membrane (both from Bio-Rad Laboratories, Hercules, CA, USA). After blocking in Tris-buffered saline containing 5\% (w/v) non-fat dry milk at room temperature for $1 \mathrm{~h}$, the membranes were incubated with specific primary antibodies overnight at $4^{\circ} \mathrm{C}$. Following washing with PBS-Tween-20, membranes were incubated with horseradish-peroxidase-conjugated secondary antibodies (Santa Cruz Biotechnology) at room temperature for $1 \mathrm{~h}$. Membranes were then incubated in ECL reagents and images were captured by Syngene Bio Imaging (Synoptics, Cambridge, UK). Densitometric quantitation of bands was performed using Syngene Bio Imaging tools.

Apoptosis assay. After exposure to experimental conditions, cells were trypsinised and incubated with PI $(1 \mu \mathrm{g} / \mathrm{ml})$ and Annexin V-FITC $(1 \mu \mathrm{g} / \mathrm{ml})$ for $15 \mathrm{~min}$ at $37^{\circ} \mathrm{C}$. Samples were then analyzed for apoptosis with a FACScan flow cytometer (Becton-Dickinson, Franklin Lakes, NJ, USA).

Measurement of intracellular ROS production. Cells were plated in black cell culture fluorometric plates at a density of $1 \times 10^{4}$ cells/well in $200 \mu \mathrm{l}$ complete medium. Each treatment was repeated in six separate wells. After cells were treated under the various experimental conditions, the cells were incubated with $100 \mu \mathrm{M}$ DCFH-DA in PBS for $30 \mathrm{~min}$ at $37^{\circ} \mathrm{C}$ in the dark followed by a PBS wash step. The fluorescence of the cells from each well was measured with a microplate reader (BioTek Synergy ${ }^{\mathrm{TM}}$ HT, USA) at $525 \mathrm{~nm}$. The percentage increase in fluorescence per well was calculated as previously described (20).

Acidic compartment detection with AO. Autophagy is characterized by formation of acidic vesicular organelles (autophagosomes and autolysosomes). AO, a fluorescent weak 
base, causes such acidic compartments to fluoresce bright red, and the cytoplasm and nucleolus to fluoresce bright green and dim green, respectively. Cells were plated on coverslips, washed in PBS with 5\% FBS and then stained with AO $(100 \mu \mathrm{g} / \mathrm{ml})$ in the dark for $15 \mathrm{~min}$ at room temperature. Staining was performed in the presence of various drugs or their vehicles, as indicated. Cells were then washed twice with PBS and analyzed under an inverted fluorescence microscope (Olympus) with a x40 objective.

Acidic compartment detection with LysoTracker Red. Cells were incubated for $15 \mathrm{~min}$ in PBS containing LysoTracker Red DND-99 (100 nM), a fluorescent acidotropic probe with a high selectivity for acidic organelles and showing good retention after aldehyde fixation. After washing with PBS, cells were fixed with $4 \%$ paraformaldehyde in PBS for $30 \mathrm{~min}$ at room temperature, washed twice with PBS, and then analyzed under the inverted fluorescence microscope (Olympus) with a $\mathrm{x} 40$ objective.

Statistical analysis. Results are expressed as means \pm standard deviation (SD) or means \pm standard error of mean (SEM), as indicated in the figure legends. Data are representative of three independent experiments performed in triplicate. Statistical analysis of the data was performed using one-way ANOVA. The Tukey's post hoc test was used to determine the significance for all pairwise comparisons of interest. Differences were considered statistically significant for values of $\mathrm{p}<0.05$.

\section{Results}

Severe ER stress induced by DTT contributes to HeLa cell death. HeLa cells were treated with increasing doses of DTT $(1.6,3.2,6.4$ and $9.6 \mathrm{mM})$ for 12 or $24 \mathrm{~h}$ and then the survival rate was detected using MTT assay. The result showed that the viability of HeLa cells was decreased by DTT in a time- and dose-dependent manner (Fig. 1A). Based on the MTT results, we treated HeLa cells with DTT (3.2 and $6.4 \mathrm{mM}$ ) for 12 or $24 \mathrm{~h}$ and then assessed apoptosis based on Hochest 33258 staining and caspase- 3 activation. Compared with the control group, apoptotic chromatin condensation was obvious in DTT-treated HeLa cells (Fig. 1B). DTT enhanced the expression of cleaved caspase-3 (Fig. 1C).

As DTT is a strong inducer of ER stress, we investigated whether ER stress was partially responsible for the cell death induced by DTT, through assessing expression of ER stress marker proteins Grp78 and Chop. Grp78 is an ER chaperone protein believed to increase the ER protein folding capacity and suppress pro-apoptotic pathways (21). Chop is the product of a gene induced by growth arrest and DNA damage, also known as the 153/C/EBP (GADD153) homology protein, involved in cell death induced by ER stress (22). We found that DTT decreased the expression of Grp78 and enhanced that of Chop (Fig. 1D). These results indicated that DTT efficiently induced intracellular apoptosis in HeLa cells, partially through an ER stress-mediated apoptotic pathway.

Previous studies showed that cell death was unavoidable only when the ER stress was too prolonged or severe (23). We observed the morphology of HeLa cells after treatment with different doses of DTT for $12 \mathrm{~h}$, followed by replace- ment with fresh medium and incubation for an additional 12 or 24 h (Fig. 1E). Compared with controls, DTT-treated cells were rounded, displaying a higher refractive index after treatment. After DTT removal, the morphology of cells previously exposed to $3.2 \mathrm{mM}$ DTT became normal, while those previously exposed to $6.4 \mathrm{mM}$ DTT remained rounded. Chop expression in cells previously exposed to $3.2 \mathrm{mM}$ DTT decreased whereas Grp78 expression remained elevated after DTT removal. In contrast, in cells previously exposed to $6.4 \mathrm{mM}$ DTT, Chop expression remained elevated whereas that of Grp78 was decreased after DTT removal (Fig. 1F). These expression patterns likely reflected cellular adaptation, including attempted restoration of ER homeostasis, occurring under milder ER stress conditions.

These results, overall, indicated that severe ER stress induced by DTT contributed to HeLa cell death.

The effect of ROS changes from pro-survival to pro-death as ER stress increased. Since DTT induces ER stress primarily by disrupting the redox state of the ER, we next investigated whether ROS were involved in DTT-induced cell death. We first assessed intracellular ROS levels in HeLa cells loaded with DCFH-DA. As shown in Fig. 2A, DTT treatment increased ROS production in HeLa cells and ROS levels were linearly correlated with DTT concentrations. Data from the MTT assay showed that NAC exacerbated cell death induced by the lower dose (3.2 mM) DTT, but attenuated that induced by $6.4 \mathrm{mM}$ DTT (Fig. 2B). The antioxidant NAC reduced the ROS levels induced by DTT (Fig. 2C).

We assessed involvement of ROS in apoptosis induced by DTT. As shown in Fig. 2D, compared with cells treated with DTT (3.2 mM) alone, cells pretreated with NAC showed enhanced expression of cleaved caspase-3. In contrast, NAC decreased cleaved caspase-3 levels in cells exposed to $6.4 \mathrm{mM}$ DTT. DTT-treated HeLa cells with or without NAC pretreatment were stained with PI and Annexin V-FITC and apoptotic cell populations quantified by flow cytometry. In cells pretreated with NAC, the percentage of apoptosis induced by $3.2 \mathrm{mM}$ DTT was increased by $17.24 \%$ ( $\mathrm{p}<0.05$; Fig. 2E). In contrast, in cells treated with $6.4 \mathrm{mM}$ DTT, NAC pretreatment decreased the apoptotic cell population by $12.56 \%$ (p<0.05; Fig. 2F).

The extent of ER stress induced by DTT was dose-dependent. Therefore, these findings indicated that ROS changed from a pro-survival to pro-death signal as the ER stress increased.

Differential activation of the MAP-kinase p38, JNK and ERK is involved in ROS-mediated cell death. The MAPKs are downstream signaling molecules activated by ROS and ER stress, and have been shown to be crucial for maintenance of cells (24). To investigate whether the MAPKs were related to the diverse roles of ROS in DTT-induced cell death, we examined activation of p38, JNK and ERK by western blotting. Low cytotoxic doses of DTT transiently activated JNK and p38, whereas high dose of DTT persistently activated JNK and p38 and simultaneously reduced ERK activity (Fig. 3A-D). Cells were next treated with DTT with or without corresponding inhibitors of JNK (SP600125), p38 (SB203580) and ERK (U0126) (Fig. 3E-G) for $24 \mathrm{~h}$. Based on the MTT assays, SP600125 or SB203580 decreased and U0126 increased, 
A

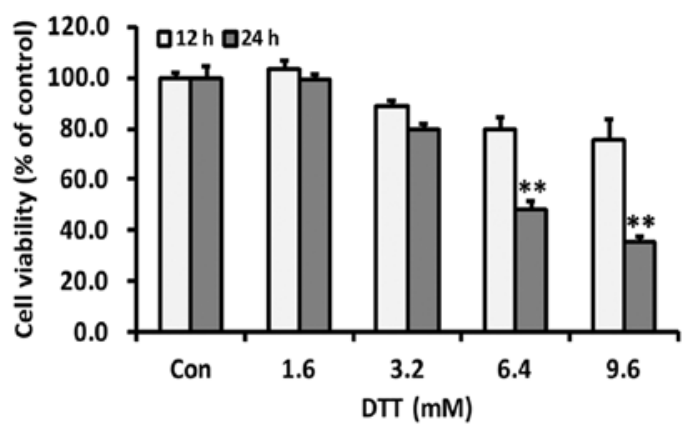

C

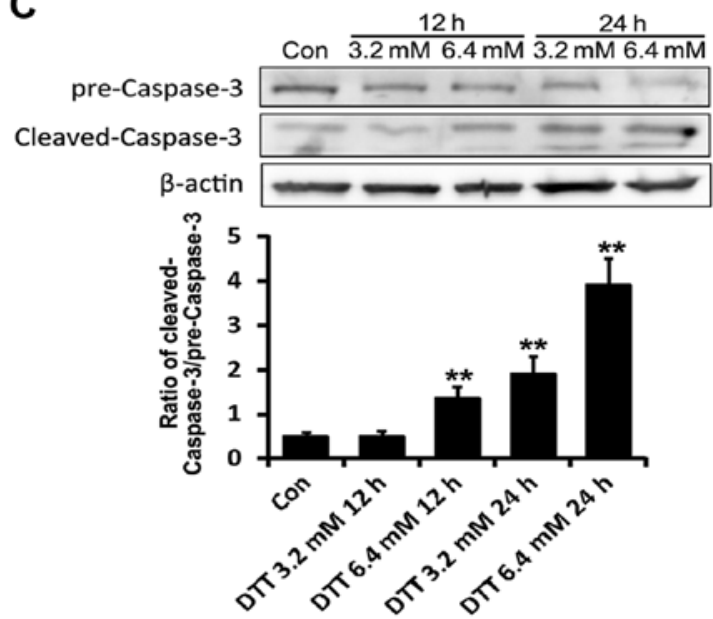

E

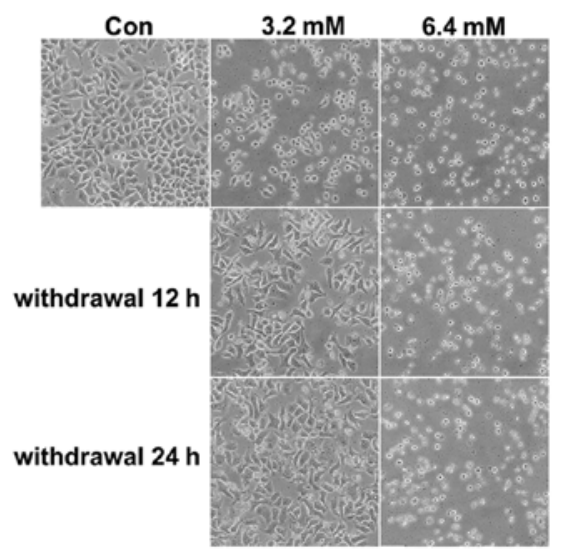

B

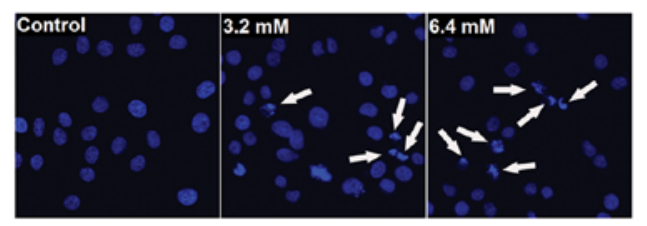

D

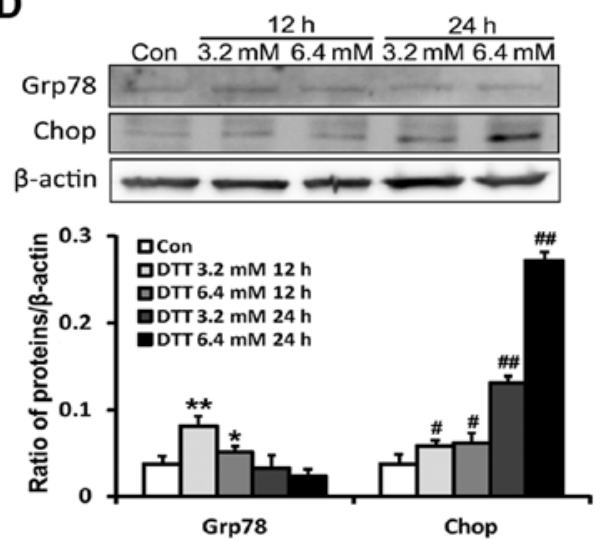

F
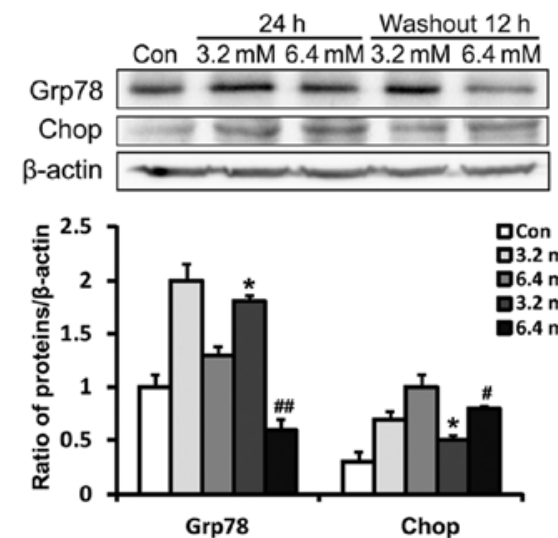

Figure 1. DTT inhibits HeLa cell growth and induces ER stress-mediated apoptosis. (A) HeLa cells were treated with DTT as indicated. Cell viability was determined by MTT assays. Data are means $\pm \mathrm{SD}, \mathrm{n}=6 ;{ }^{* *} \mathrm{p}<0.01$ vs. the $12 \mathrm{~h}$ group. (B) HeLa cells were treated with DTT for $24 \mathrm{~h}$ and stained with Hochest 33258. Cell morphology was observed by fluorescent microscopy (bar indicates $20 \mu \mathrm{m}$; arrows indicate apoptotic cells). (C and D) Western blot analysis for the expression of caspase-3, cleaved caspase-3, Grp78 and Chop, in HeLa cells treated with DTT for 12 or $24 \mathrm{~h}$. The ratio of cleaved caspase-3 to total caspase-3 (cleaved caspase-3/caspase-3) is shown. Quantitation of Grp78, Chop and caspase-3 protein levels. Data are means \pm SD, $n=3$; " $p<0.05$ vs. the control group; ${ }^{* *} \mathrm{p}<0.01$ vs. the control group. $(\mathrm{E}$ and F) HeLa cells were treated with DTT for $24 \mathrm{~h}$ and DTT was then removed and replaced with fresh medium. Cell morphology was observed by inverted microscopy at a magnification of x200. Western blot analysis for the expression of Grp78 and Chop. Quantitation of Grp78, Chop protein levels. Data are means $\pm \mathrm{SD}, \mathrm{n}=3$; ${ }^{*} \mathrm{p}<0.05$ vs. $3.2 \mathrm{mM}$ DTT group; ${ }^{\#} \mathrm{p}<0.05$ vs. $6.4 \mathrm{mM}$ DTT group; ${ }^{\# \#}$ p $<0.01$ vs. $6.4 \mathrm{mM}$ DTT group.

DTT-induced cytotoxity in HeLa cells. The inhibitors alone exhibited no significant cytotoxity.

These results suggested that DTT differentially activated the MAPK signaling pathways in a dose-dependent manner, with this balance determining the final fate of the cells.

Severe ER stress inhibits autophagic flux. Previous studies showed that ROS and ER stress may regulate autophagy through the MAPKs $(25,26)$. We examined whether the role of autophagy changed when the ER stress increased. Through detecting expressions of the autophagy markers p62 and
LC3-II, we found that DTT activated autophagy (Fig. 4A). After DTT removal, p62 expression decreased in cells previously exposed to $3.2 \mathrm{mM}$ DTT, while it persistently increased in those previously exposed to $6.4 \mathrm{mM}$ DTT (Fig. 4B). Since p62 is one of the substrate proteins degraded through autophagy, its expression is an indicator of autophagy flux $(27,28)$. These data implied that autophagy flux changed with increased ER stress. Therefore, we examined autophagy flux further by assessing p62 expression in cells treated with DTT in combination with the autophagy inhibitor chloroquine (CQ) (Fig. 4C). CQ, which inhibits fusion of autophagosomes 
A

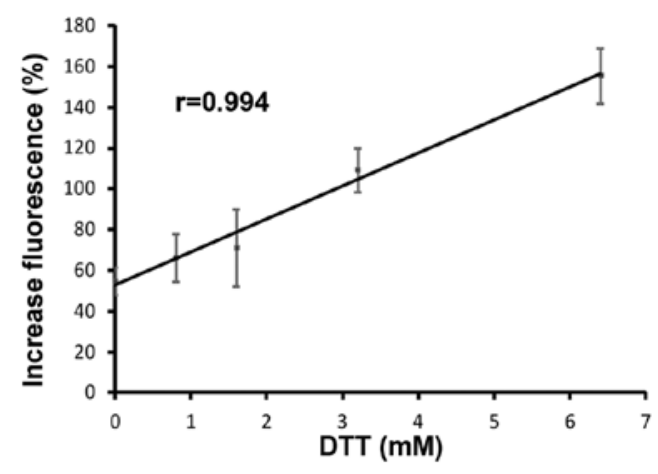

B

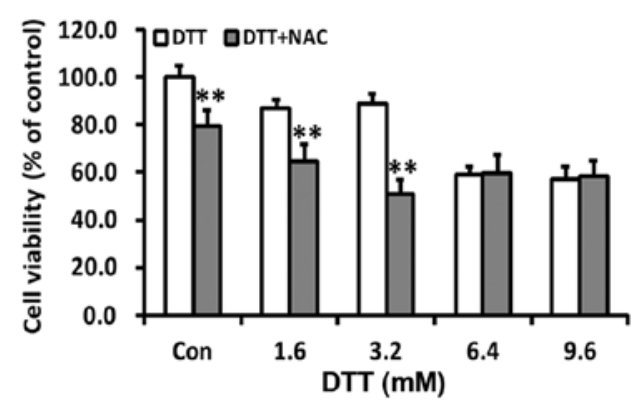

C

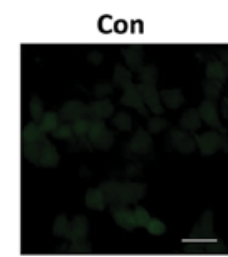

$3.2 \mathrm{mM}$

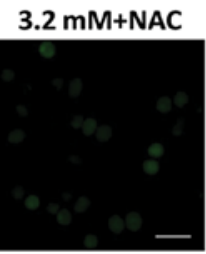

$6.4 \mathrm{mM}$

6.4 $\mathrm{mM}+\mathrm{NAC}$
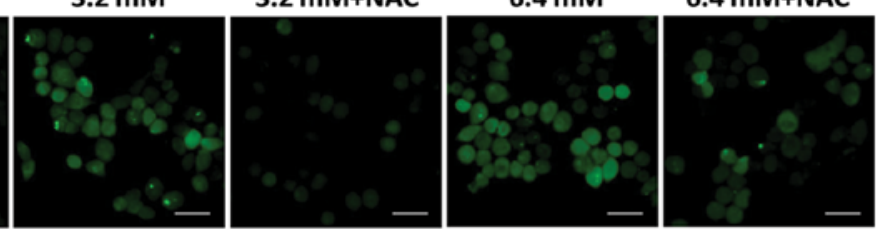

D

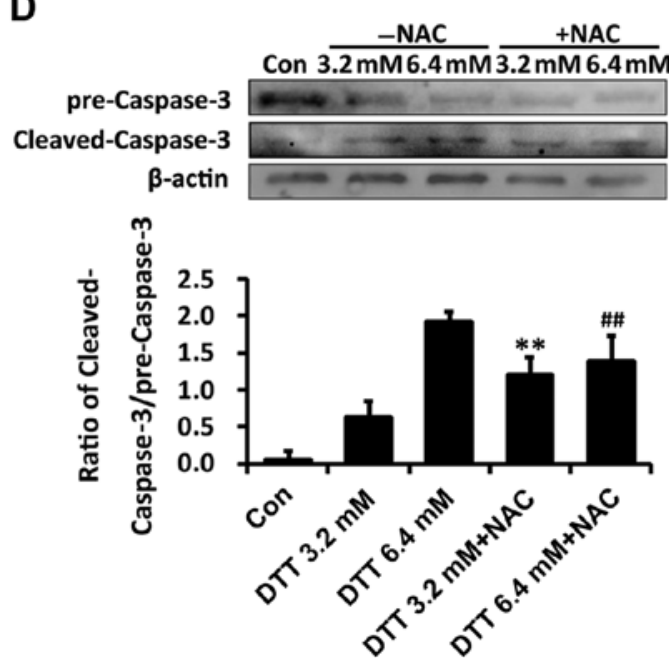

E
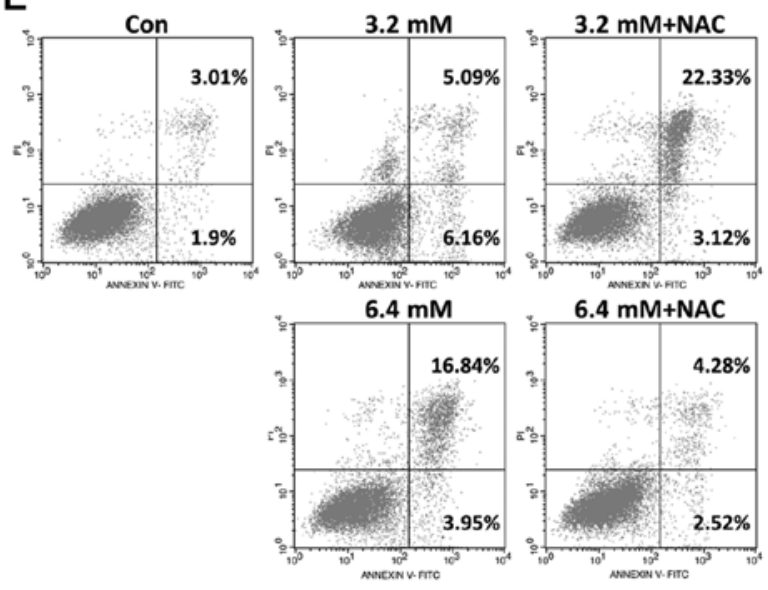

Figure 2. ROS involvement in DTT-induced apoptosis. (A) Concentration-dependent increases in DCF fluorescence in HeLa cells induced by $12 \mathrm{~h}$ exposure to DTT as indicated. Each data point is the mean of data from 6-wells $(\mathrm{n}=6)$ with error bars representing SEM. The line was created by linear regression and $\mathrm{r}$ is the correlation coefficient. (B) HeLa cells were treated with DTT with or without NAC $(100 \mu \mathrm{M})$ for $24 \mathrm{~h}$. Cell viability was determined with the MTT assay. Data are means $\pm \mathrm{SD}, \mathrm{n}=6$; ${ }^{* *} \mathrm{p}<0.01$ vs. DTT. (C) Fluorescence in DCFH-DA-loaded cells was observed by fluorescence microscopy (bar indicates $20 \mu \mathrm{m}$ ). (D) Western blot analyses showing expression of caspase-3 and cleaved caspase-3. The ratio of cleaved caspase-3 to total caspase-3 (cleaved caspase-3/caspase-3) is shown. Data are means $\pm \mathrm{SD}, \mathrm{n}=3 ;{ }^{* *} \mathrm{p}<0.01$ vs. $3.2 \mathrm{mM}$ DTT group; ${ }^{\# \#} \mathrm{p}<0.01$ vs. $6.4 \mathrm{mM}$ DTT group. (E) Cells were stained with PI and Annexin V-FITC. The positively stained cells were counted using the FACScan. Data are means $\pm \mathrm{SD}, \mathrm{n}=3$.

and lysosomes, enhanced the p62 expression induced by DTT. However, the increase in p62 in cells exposed to DTT with CQ was negatively correlated with DTT dose. Our explanation is that the autophagy flux in cells exposed to the lower dose of DTT was relatively normal and efficient, whereas that in cells exposed to the higher dose was partially blocked. To check the role of autophagy in DTT-induced cell death, we used the compound 3-MA, which inhibits initiation of autophagy. Based on the MTT assay, 3-MA enhanced the cell death induced by DTT (Fig. 4D). We next detected cleaved caspase-3, as an indicator of apoptosis activation. 3-MA enhanced the expression of cleaved caspase-3 induced by DTT in HeLa cells (Fig. 4E).

These results indicated that, in HeLa cells, autophagy flux was partially blocked during severe ER stress and that inhibition of autophagy increased cytotoxicity induced by DTT.
Downregulated ERK phosphorylation mediates inhibition of autophagic flux. To elucidate whether blocked autophagy flux was the result of downregulated ERK phosphorylation, controlled by ROS, we first examined effects of ROS on autophagy flux and ERK activation. As shown in Fig. 5A, compared with cells exposed to $3.2 \mathrm{mM}$ DTT alone, cells exposed to $3.2 \mathrm{mM}$ DTT with NAC had decreased p-ERK and increased p62 expression. In contrast, p-ERK expression in cells exposed to $6.4 \mathrm{mM}$ DTT was further increased, while that of p62 was decreased, after pretreatment with NAC. This indicated that ROS inactivated the ERK signaling pathway and blocked the autophagy flux when the stress was severe.

To determine whether ERK is involved in regulating autophagy flux, we monitored p62 expression in cells treated with DTT and the ERK inhibitor U0126. As shown in Fig. 5B, 
A
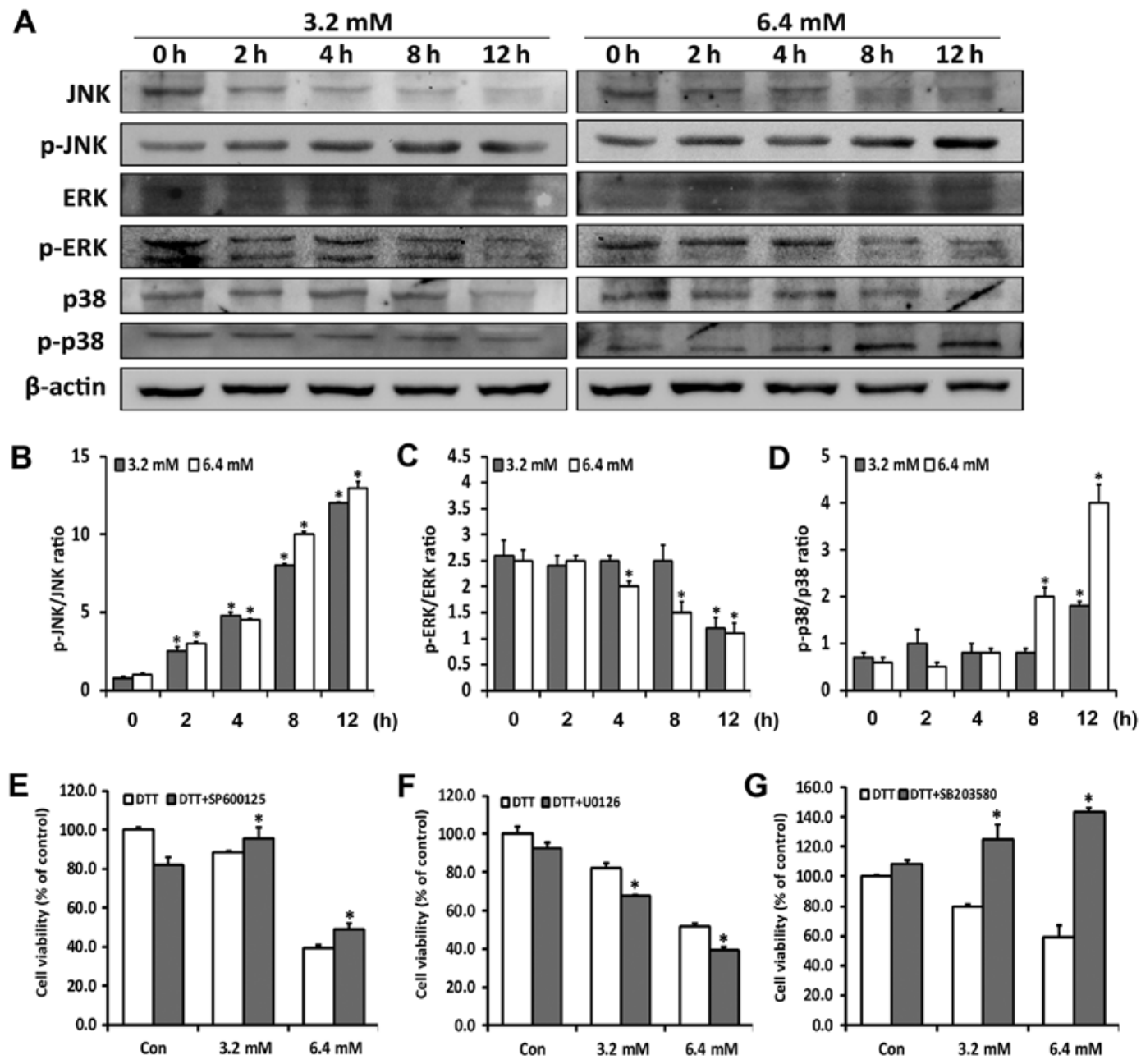

Figure 3. ROS induces apoptosis through differentially activation of JNK, p38 and ERK pathways in HeLa cells. (A-D) Protein expression levels were detected by western blotting following DTT treatment for various times $(0-12 \mathrm{~h})$. Quantitation of $\mathrm{p}-\mathrm{JNK}, \mathrm{p}-\mathrm{ERK}$ and $\mathrm{p}$-p38 protein levels. Data are means $\pm \mathrm{SD}$, $\mathrm{n}=3$; ${ }^{*} \mathrm{p}<0.05$ vs. Con. (E-G) HeLa cells were treated with DTT with or without the following inhibitors: SP600125 (10 $\left.\mu \mathrm{M}\right), \mathrm{U} 0126$ (10 $\left.\mu \mathrm{M}\right)$ or SB203580 (10 $\left.\mu \mathrm{M}\right)$ for $24 \mathrm{~h}$. Cell viability was determined with the MTT assay.

compared with cells exposed to DTT alone, cells exposed to DTT with U0126 had higher p62 expression. However, the increase in p62 expression in the presence of U0126 was inversely correlated with DTT dose. Similar to that observed in cells treated with DTT and CQ, U0126 changed the expression profile of p62 induced by DTT. This indicated that ERK inactivation led to blockade of autophagy flux. We next monitored accumulation of cellular acidic compartments in cells treated by DTT with or without CQ or U0126, using LysoTracker Red and AO staining. The lysosome specific stain LysoTracker Red and the $\mathrm{pH}$-sensitive probe $\mathrm{AO}$ have been frequently used to detect autophagic flux (29). In cells stained with LysoTracker Red, the red-dotted structures are lysosomes or structures formed by fusion of autophagosomes and lysosomes. In cells stained with $\mathrm{AO}$, red fluorescence indicates acid vesicular structures such as lysosomes and fused autophagosomes. As shown in Fig. 5C and D, compared with cells exposed to DTT alone, cells exposed to DTT with CQ or U0126 showed clearly increased numbers of red-dotted structures and enhanced red fluorescence. These observations indicated that U0126 and CQ similarly increased accumulation of cellular acidic components induced by DTT.
These results indicated that ERK inhibition during severe ER stress contributed to blockade of autophagy flux by affecting the fusion between autophagosomes and lysosomes.

\section{Discussion}

Numerous studies have explored the relationship between ER stress and autophagy. However, the signaling pathways responsible, during ER stress, for the outcome of life-or-death in autophagy appear to vary with cell types and stimuli. Our study explored the roles of ROS and downstream events in the autophagy and apoptosis that follow DTT-triggered ER stress in HeLa cells. Our results indicated that: i) severe ER stress induced by DTT contributes to cell death, ii) the role of ROS changes from pro-survival to pro-death with increased ER stress, iii) differential activation of the MAP kinases $\mathrm{p} 38$ and ERK are involved in ROS-mediated cell death and iv) ERK phosphorylation inhibited by ROS is involved in blockade of autophagy flux.

Consistent with the results we report in this study, the extent and duration of ER stress is often regarded as closely related to the final fate of the cells (30). Our results confirmed 
A

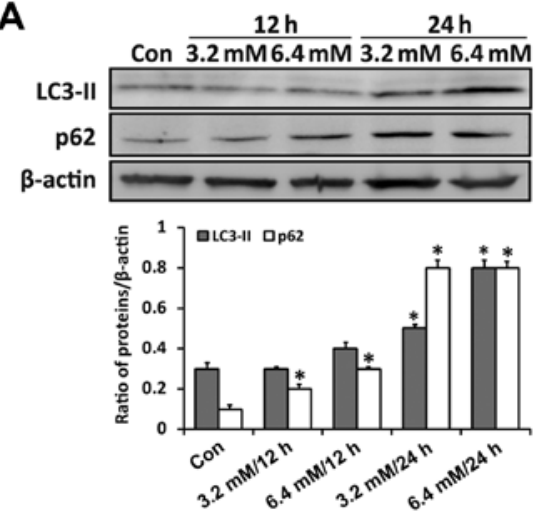

C
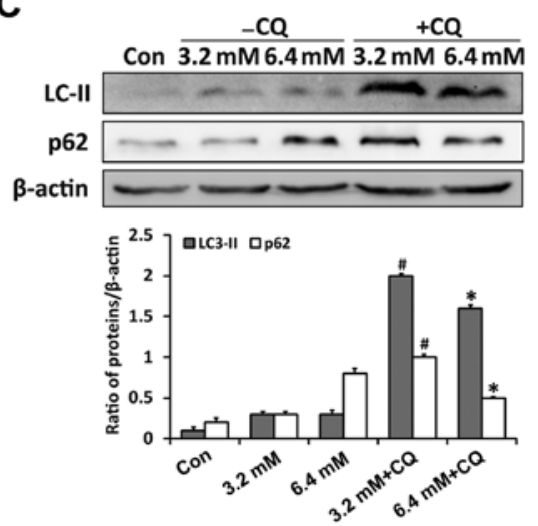

B

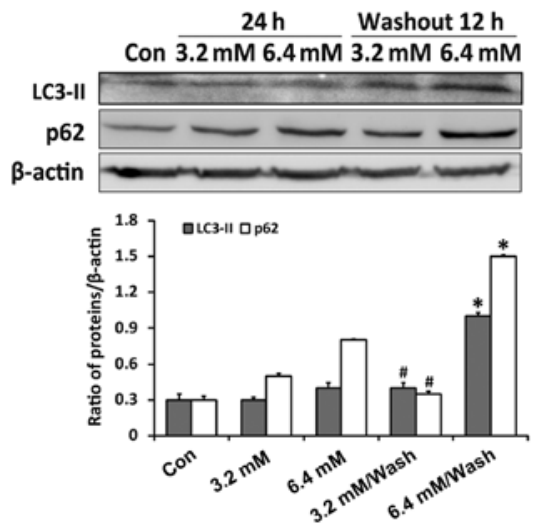

E
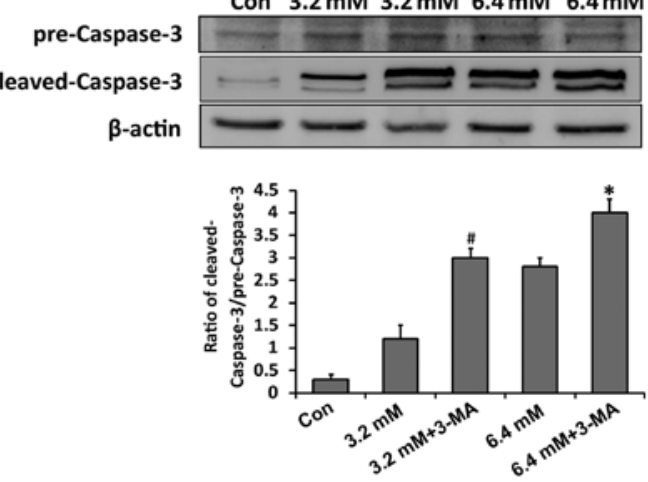

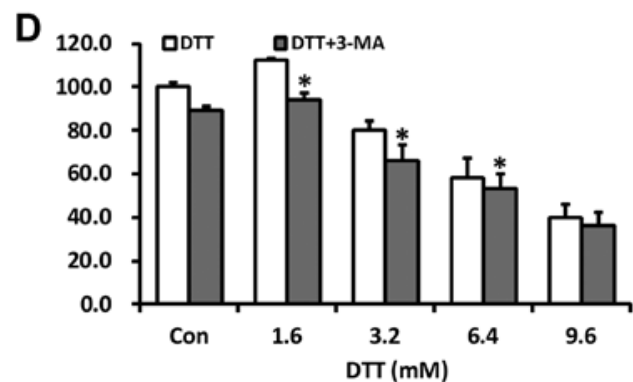

Figure 4. DTT induces autophagy in HeLa cells. Western blot analyses showing expression of LC3-II and p62 proteins. (A) HeLa cells were treated with DTT for 12 or $24 \mathrm{~h}$. Quantitation of LC3-II and p62 protein levels. Data are means \pm SD, n=3; ${ }^{*}$ p $<0.05$ vs. Con. (B) HeLa cells were treated with DTT for $24 \mathrm{~h}$ then DTT removed with fresh medium replacement. (C) HeLa cells were treated with DTT with or without CQ (50 $\mu \mathrm{M})$ for $24 \mathrm{~h}$. Quantitation of LC3-II and p62 protein levels. Data are means $\pm \mathrm{SD}, \mathrm{n}=3 ;{ }^{*} \mathrm{p}<0.05$ vs. $3.2 \mathrm{mM}$ DTT group; ${ }^{*} \mathrm{p}<0.05$ vs. $6.4 \mathrm{mM}$ DTT group. (D and E) HeLa cells were treated with DTT with or without 3-MA $(10 \mathrm{mM})$ for $24 \mathrm{~h}$. Cell viability was determined with the MTT assay. Data are means $\pm \mathrm{SD}, \mathrm{n}=6$; ${ }^{*} \mathrm{p}<0.05 \mathrm{vs}$. DTT. Western blot analysis showing caspase- 3 and cleaved caspase-3. The ratio of cleaved caspase-3 to total caspase-3 (cleaved caspase-3/pre-caspase-3) is shown. Data are means \pm SD, $\mathrm{n}=3$; ${ }^{\text {p }}<<0.05$ vs. $3.2 \mathrm{mM}$ DTT group; " $\mathrm{p}<0.05$ vs. $6.4 \mathrm{mM}$ DTT group.

that human cervical cancer or HeLa cells could, partially or fully, restore ER homeostasis after exposure to low concentrations of DTT while, with exposure to higher concentrations, they continued to be stressed. The apoptosis induced by DTT in HL-60 cells was associated with early caspase-3 activation and was independent of mitochondria (31). To understand whether the DTT-induced cell damage in HeLa cells was related to over-extended ER stress, we investigated expression of Grp78 and Chop, whose levels are hallmarks of ER stress. Downregulation of Grp78 with elevated Chop expression exacerbated retinal deterioration and apoptosis during retinal ischemia/reperfusion (I/R) injury (32). Our findings were consistent with this report. During mild ER stress, cells that displayed higher Grp78 and lower Chop expression might adapt to the stress. After withdrawal of the stressor, cells with relatively low Grp78 and high Chop expression would ultimately undergo apoptosis. Our results suggested that the severe ER stress induced by DTT contributed to cell death in HeLa cells.

ROS were involved in DTT-induced cell death under severe ER stress. Hydrogen peroxide $\left(\mathrm{H}_{2} \mathrm{O}_{2}\right)$ is generated as a by-product in the ER during disulfide bond formation driven by the Ero1/PDI system (33). Schuiki et al reported that DTT quickly and potently reduced the normally oxidizing ER environment (1). This implies that oxidative stress was upstream in DTT-exposed cells and ROS may have determined the final impact of ER stress on cell fate. In HT1080 cells treated with DTT, $\mathrm{H}_{2} \mathrm{O}_{2}$ in the ER lumen was markedly increased, leading to peroxiredoxin IV (PrxIV) hyperoxidation (33). In our study, we found that $\mathrm{H}_{2} \mathrm{O}_{2}$ production was increased in DTT-treated HeLa cells in a DTT dose-dependent manner. Removal of ROS with the antioxidant NAC promoted DTT-induced 
A
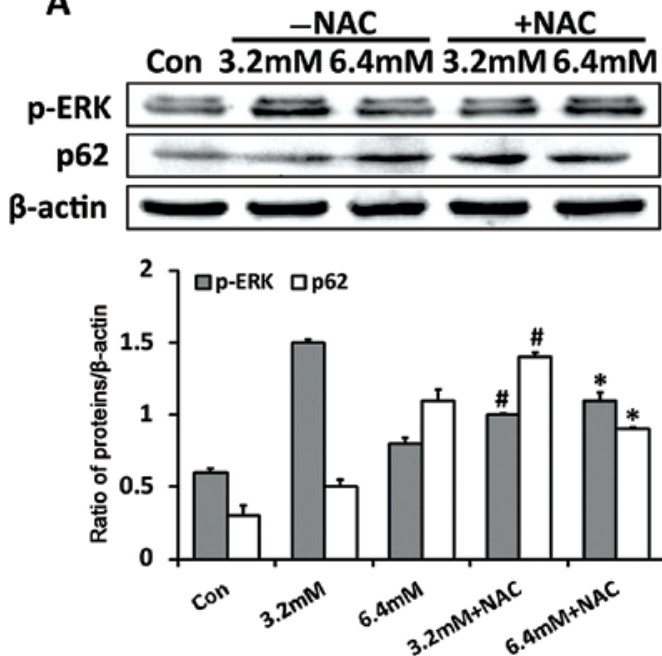

C

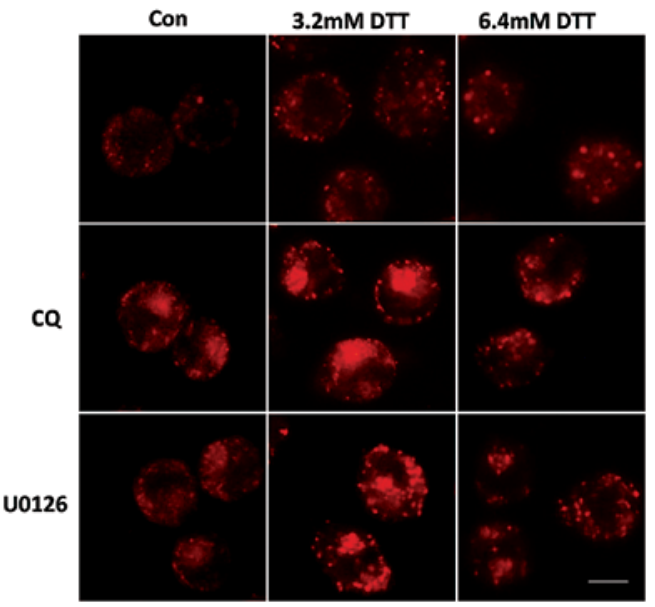

B
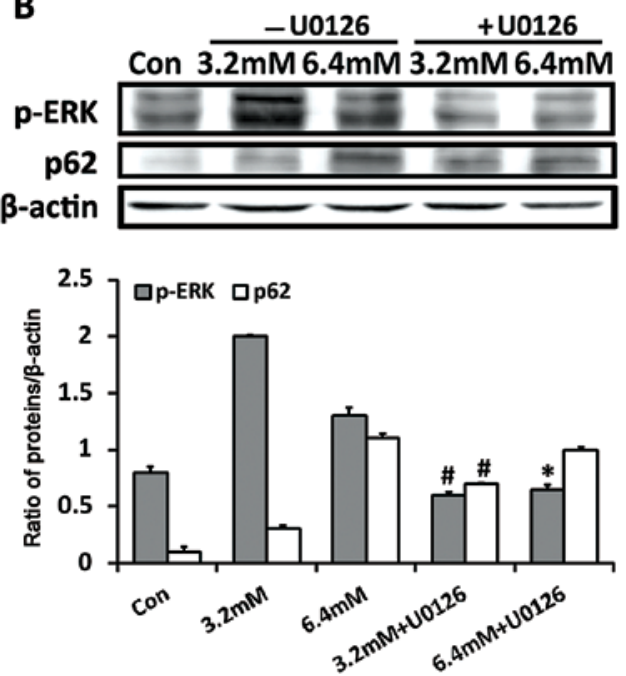

D

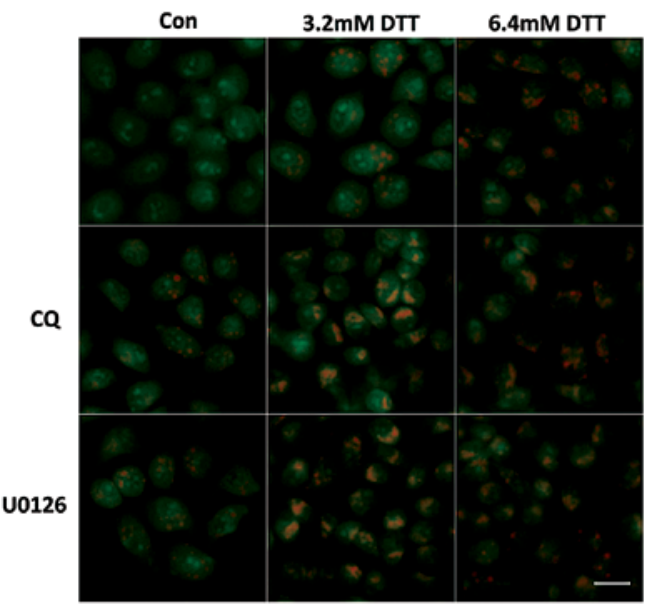

Figure 5. ERK links the DTT-induced ROS increases and inhibition of autophagy flux. HeLa cells were treated with DTT with or without NAC (100 $\mu$ M) or U0126 $(10 \mu \mathrm{M})$ for $12 \mathrm{~h}$. (A and B) Western blot analyses showing expression of p-ERK and p62. Quantitation of p-ERK and p62 protein levels. Data are means $\pm \mathrm{SD}, \mathrm{n}=3$; ${ }^{\#} \mathrm{p}<0.05$ vs. $3.2 \mathrm{mM}$ DTT group; ${ }^{*} \mathrm{p}<0.05$ vs. $6.4 \mathrm{mM}$ DTT group. (C and D) HeLa cells were treated with DTT (3.2 or 6.4 mM) with or without CQ $(50 \mu \mathrm{M})$ or U0126 $(10 \mu \mathrm{M})$ for $24 \mathrm{~h}$. Representative micrographs of LysoTracker Red stained HeLa cells (magnification, $\mathrm{x} 40)$ and representative micrographs of AO stained HeLa cells (magnification, x20).

cytotoxity under milder ER stress, but attenuated its effects when ER stress was severe. Our results are consistent with the concept that intracellular ROS levels at any moment can determine the fate of the cells. Low levels of ROS function as regulators of cell signaling while accumulation of higher ROS levels induces apoptosis $(34,35)$. The implication is that a prolonged $\mathrm{H}_{2} \mathrm{O}_{2}$ signal is responsible for shifting the outcome of ER stress from cell survival to cell death.

Activation of the ASK1-JNK/p38 pathway, among the signaling pathways responsible for apoptosis following ER stress was dependent on $\operatorname{ROS}(36,37)$. In Eca109 cells, p38 MAPK kinase was essential for DTT- and cisplatin-induced apoptosis (38). Our results showed that JNK and p38 activation persisted with increased ER stress, while ERK activation was attenuated early in HeLa cells undergoing prolonged exposure to DTT. In gastric cancer cells, MEK inhibition blocked Grp78 upregulation and enhanced apoptosis induced by ER stress (8). Our results showed that JNK/p38 inhibition attenuated DTT-induced cytotoxity while ERK inhibition promoted DTT-induced cell death. These results indicated that differential activation of MAPK pathways occurred during various extent of ER stress and this balance may determine the final outcome of the cells.

Autophagy is important for alleviating ER stress (39). Numerous studies have indicated that ER stress was induced simultaneously with activation of autophagy $(40,41)$. Our findings suggested that the autophagy flux decreased with increased ER stress. ROS removal with NAC simultaneously affected ERK activation and autophagy flux. Previous studies showed that ERK activation was involved in the process of autophagosome and lysosome fusion (19). Our data demonstrated that inhibition of ERK affected autophagy flux in a similar manner as did the classic autophagy inhibitor CQ, which inhibits the fusion process of autophagosome and lysosome. When the autophagy pathway was inhibited by the specific inhibitor 3-MA, DTT-induced cytotoxity was increased. These results indicated that autophagy flux was negatively correlated with the extent of ER stress and that ROS blocked autophagy flux through inhibiting ERK, potentially contributing to cell death during severe ER stress.

As ER stress and autophagy are targets for cancer therapy (42), there is a need for more research to explore the 
mechanisms linking these two processes. Our findings indicate that the MAPKs are vital to regulating autophagy during ER-associated oxidative stress. ROS may control autophagy flux through regulating ERK phosphorylation.

\section{Acknowledgements}

The present study was supported by the National Natural Science Foundation of China (nos. 81272876 and 81202552).

\section{References}

1. Schuiki I, Zhang L and Volchuk A: Endoplasmic reticulum redox state is not perturbed by pharmacological or pathological endoplasmic reticulum stress in live pancreatic $\beta$-cells. PLoS One 7: e48626, 2012.

2. Bhandary B, Marahatta A, Kim HR and Chae HJ: An involvement of oxidative stress in endoplasmic reticulum stress and its associated diseases. Int J Mol Sci 14: 434-456, 2012.

3. Cameron NE: Role of endoplasmic reticulum stress in diabetic neuropathy. Diabetes 62: 696-697, 2013.

4. Mota SI, Costa RO, Ferreira IL, Santana I, Caldeira GL, Padovano C, Fonseca AC, Baldeiras I, Cunha C, Letra L, et al: Oxidative stress involving changes in Nrf2 and ER stress in early stages of Alzheimer's disease. Biochim Biophys Acta 1852: $1428-1441,2015$

5. Yadav RK, Chae SW, Kim HR and Chae HJ: Endoplasmic reticulum stress and cancer. J Cancer Prev 19: 75-88, 2014.

6. Sui X, Kong N, Ye L, Han W, Zhou J, Zhang Q, He C and Pan H: p38 and JNK MAPK pathways control the balance of apoptosis and autophagy in response to chemotherapeutic agents. Cancer Lett 344: 174-179, 2014.

7. Matsuzawa A and Ichijo H: Redox control of cell fate by MAP kinase: Physiological roles of ASK1-MAP kinase pathway in stress signaling. Biochim Biophys Acta 1780: 1325-1336, 2008.

8. Zhang LJ, Chen S, Wu P, Hu CS, Thorne RF, Luo CM, Hersey P and Zhang XD: Inhibition of MEK blocks GRP78 up-regulation and enhances apoptosis induced by ER stress in gastric cancer cells. Cancer Lett 274: 40-46, 2009.

9. Han J, Back SH, Hur J, Lin YH, Gildersleeve R, Shan J, Yuan CL, Krokowski D, Wang S, Hatzoglou M, et al: ER-stress-induced transcriptional regulation increases protein synthesis leading to cell death. Nat Cell Biol 15: 481-490, 2013.

10. Son Y, Cheong YK, Kim NH, Chung HT, Kang DG and Pae HO Mitogen-activated protein kinases and reactive oxygen species: How can ROS activate MAPK pathways? J Signal Transduct 2011: 792639, 2011.

11. Glick D, Barth S and Macleod KF: Autophagy: Cellular and molecular mechanisms. J Pathol 221: 3-12, 2010.

12. Yorimitsu T, Nair U, Yang Z and Klionsky DJ: Endoplasmic reticulum stress triggers autophagy. J Biol Chem 281: 30299-30304, 2006

13. Ogata M, Hino S, Saito A, Morikawa K, Kondo S, Kanemoto S Murakami T, Taniguchi M, Tanii I, Yoshinaga K, et al: Autophagy is activated for cell survival after endoplasmic reticulum stress. Mol Cell Biol 26: 9220-9231, 2006.

14. Ding WX, Ni HM, Gao W, Hou YF, Melan MA, Chen X, Stolz DB, Shao ZM and Yin XM: Differential effects of endoplasmic reticulum stress-induced autophagy on cell survival. J Biol Chem 282: 4702-4710, 2007.

15. Høyer-Hansen M and Jäättelä M: Connecting endoplasmic reticulum stress to autophagy by unfolded protein response and calcium. Cell Death Differ 14: 1576-1582, 2007.

16. Sridharan S, Jain K and Basu A: Regulation of autophagy by kinases. Cancers 3: 2630-2654, 2011.

17. Li C and Johnson DE: Bortezomib induces autophagy in head and neck squamous cell carcinoma cells via JNK activation. Cancer Lett 314: 102-107, 2012.

18. Haberzettl P and Hill BG: Oxidized lipids activate autophagy in a JNK-dependent manner by stimulating the endoplasmic reticulum stress response. Redox Biol 1: 56-64, 2013.

19. Kao C, Chao A, Tsai CL, Chuang WC, Huang WP, Chen GC, Lin CY, Wang TH, Wang HS and Lai CH: Bortezomib enhances cancer cell death by blocking the autophagic flux through stimulating ERK phosphorylation. Cell Death Dis 5: e1510, 2014.
20. Wang $\mathrm{H}$ and Joseph JA: Quantifying cellular oxidative stress by dichlorofluorescein assay using microplate reader. Free Radic Biol Med 27: 612-616, 1999.

21. Luo B and Lee AS: The critical roles of endoplasmic reticulum chaperones and unfolded protein response in tumorigenesis and anticancer therapies. Oncogene 32: 805-818, 2013.

22. Marciniak SJ, Yun CY, Oyadomari S, Novoa I, Zhang Y, Jungreis R, Nagata K, Harding HP and Ron D: CHOP induces death by promoting protein synthesis and oxidation in the stressed endoplasmic reticulum. Genes Dev 18: 3066-3077, 2004.

23. Rubio C, Pincus D, Korennykh A, Schuck S, El-Samad H and Walter P: Homeostatic adaptation to endoplasmic reticulum stress depends on Ire1 kinase activity. J Cell Biol 193: 171-184, 2011.

24. Rutkowski DT and Kaufman RJ: That which does not kill me makes me stronger: Adapting to chronic ER stress. Trends Biochem Sci 32: 469-476, 2007.

25. Scherz-Shouval R and Elazar Z: Regulation of autophagy by ROS: Physiology and pathology. Trends Biochem Sci 36: 30-38, 2011.

26. Matsumoto H, Miyazaki S, Matsuyama S, Takeda M, Kawano M, Nakagawa H, Nishimura K and Matsuo S: Selection of autophagy or apoptosis in cells exposed to ER-stress depends on ATF4 expression pattern with or without CHOP expression. Biol Open 2: 1084-1090, 2013.

27. Komatsu M and Ichimura Y: Physiological significance of selective degradation of p62 by autophagy. FEBS Lett 584: 1374-1378, 2010

28. Yang X, Xiang X, Xia M, Su J, Wu Y, Shen L, Xu Y and Sun L: Inhibition of JNK3 promotes apoptosis induced by BH3 mimetic $\mathrm{S} 1$ in chemoresistant human ovarian cancer cells. Anat Rec 298: 386-395, 2015.

29. Degtyarev M, Reichelt M and Lin K: Novel quantitative autophagy analysis by organelle flow cytometry after cell sonication. PLoS One 9: e87707, 2014.

30. Hetz C: The unfolded protein response: Controlling cell fate decisions under ER stress and beyond. Nat Rev Mol Cell Biol 13: 89-102, 2012

31. Tartier L, McCarey YL, Biaglow JE, Kochevar IE and Held KD: Apoptosis induced by dithiothreitol in HL-60 cells shows early activation of caspase 3 and is independent of mitochondria. Cell Death Differ 7: 1002-1010, 2000.

32. Li H, Zhu X, Fang F, Jiang D and Tang L: Down-regulation of GRP78 enhances apoptosis via CHOP pathway in retinal ischemia-reperfusion injury. Neurosci Lett 575: 68-73, 2014.

33. Tavender TJ and Bulleid NJ: Peroxiredoxin IV protects cells from oxidative stress by removing $\mathrm{H}_{2} \mathrm{O}_{2}$ produced during disulphide formation. J Cell Sci 123: 2672-2679, 2010.

34. Fleury C, Mignotte B and Vayssière JL: Mitochondrial reactive oxygen species in cell death signaling. Biochimie 84: 131-141, 2002.

35. Scherz-Shouval R and Elazar Z: ROS, mitochondria and the regulation of autophagy. Trends Cell Biol 17: 422-427, 2007.

36. Noguchi T, Ishii K, Fukutomi H, Naguro I, Matsuzawa A, Takeda $\mathrm{K}$ and Ichijo $\mathrm{H}$ : Requirement of reactive oxygen species-dependent activation of ASK1-p38 MAPK pathway for extracellular ATP-induced apoptosis in macrophage. J Biol Chem 283: 7657-7665, 2008.

37. Shore GC, Papa FR and Oakes SA: Signaling cell death from the endoplasmic reticulum stress response. Curr Opin Cell Biol 23: 143-149, 2011.

38. Zhang QX, Feng R, Zhang W, Ding Y, Yang JY and Liu GH: Role of stress-activated MAP kinase P38 in cisplatin- and DTT-induced apoptosis of the esophageal carcinoma cell line Eca109. World J Gastroenterol 11: 4451-4456, 2005.

39. Kroemer G, Mariño G and Levine B: Autophagy and the integrated stress response. Mol Cell 40: 280-293, 2010.

40. Kapuy O, Vinod PK and Bánhegyi G: mTOR inhibition increases cell viability via autophagy induction during endoplasmic reticulum stress - An experimental and modeling study. FEBS Open Bio 4: 704-713, 2014.

41. Xi H, Kurtoglu M, Liu H, Wangpaichitr M, You M, Liu X, Savaraj $\mathrm{N}$ and Lampidis TJ: 2-Deoxy-D-glucose activates autophagy via endoplasmic reticulum stress rather than ATP depletion. Cancer Chemother Pharmacol 67: 899-910, 2011.

42. Verfaillie T, Salazar M, Velasco G and Agostinis P: Linking ER stress to autophagy: Potential implications for cancer therapy. Int J Cell Biol 2010: 930509, 2010. 